\title{
Surgical management of incidentally discovered diffusely infiltrating low-grade glioma
}

\author{
Michael Opoku-Darko, MD, MSc, ${ }^{1}$ Stefan T. Lang, MD, ${ }^{1}$ James Artindale, BSc, PEng, ${ }^{4}$ \\ J. Gregory Cairncross, MD, ${ }^{2,3}$ Robert J. Sevick, MD, ${ }^{4}$ and John J. P. Kelly, MD, PhD ${ }^{1,2}$ \\ ${ }^{1}$ Division of Neurosurgery, ${ }^{2}$ The Arne Charbonneau Cancer Institute, and Departments of ${ }^{3} \mathrm{Clinical}$ Neurosciences and \\ ${ }^{4}$ Diagnostic Imaging, University of Calgary, Alberta, Canada
}

\begin{abstract}
OBJECTIVE Occasionally, diffusely infiltrating low-grade gliomas (LGGs) are identified as incidental findings in patients who have no signs or symptoms that can be ascribed to the tumors. The diagnosis of incidental, asymptomatic LGGs has become more frequent due to the vast increase in access to medical imaging technology. While management of these lesions remains controversial, early surgery has been suggested to improve outcome. The authors set out to identify and review the characteristics and surgical outcomes of patients who underwent surgical intervention for incidental LGG.

METHODS All cases of LGG surgically treated between 2004 and 2016 at the authors' institution were analyzed to identify those that were discovered incidentally. Patients with incidentally discovered LGGs were identified, and their cases were retrospectively reviewed. An "incidental" finding was defined as an abnormality on imaging that was obtained for a reason not attributable to the glioma, such as trauma, headache, screening, or research participation. Kaplan-Meier analysis was performed to determine actuarial rates of overall survival, progression-free survival, and malignant progression-free survival.
\end{abstract}

RESULTS In 34 (6.8\%) of 501 adult patients who underwent surgery for LGG, the tumors were discovered incidentally. Headache $(26 \%, n=9)$ and screening $(21 \%, n=7)$ were the most common indications for brain imaging in this group. Four of these 34 patients had initial biopsy after the tumor was identified on imaging. In 5 cases, the patients opted for immediate resection; the remaining cases were managed with a "watch-and-wait" approach, with intervention undertaken only after radiological or clinical evidence of disease progression. The mean duration of follow-up for all 34 cases was 5 years. Twelve patients (35.3\%) had disease progression, with an average time to progression of 43.8 months (range 3-105 months). There were 5 cases (14.7\%) of malignant progression and 4 deaths (11.8\%). Oligodendroglioma was diagnosed in 16 cases (47\%) and astrocytoma in 15 (44\%). Twenty-five patients (74\%) had IDH1 mutation and demonstrated prolonged survival. Only 2 patients had mild surgery-related complications, and 16 patients (47\%) developed epilepsy during the course of the disease.

CONCLUSIONS In this retrospective analysis of cases of incidentally discovered LGGs, the tumors were surgically removed with minimal surgical risk. In patients with incidental LGGs there is improved overall survival relative to median survival for patients with symptomatic LGGS, which is likely attributable to the underlying favorable biology of the disease indicated by the presence of IDH1 mutation in $74 \%$ of the cases.

https://thejns.org/doi/abs/10.3171/2017.3.JNS17159

KEY WORDS low-grade glioma; incidental; asymptomatic; survival; oncology

I NCIDENTALLY discovered brain lesions are identified as imaging abnormalities detected in patients who undergo brain imaging for an unrelated medical issue. The likelihood of encountering such "incidentalomas" is rising due in part to advances in imaging technology and the increasing number of people around the world who have access to modern diagnostic imaging. The incidence of incidental findings on brain imaging ranges from $0.05 \%$ to $3 \%$ among the healthy adult population. . $^{213,17,18,21,26}$ These incidental findings may be a consequence of radiological screening of asymptomatic patients for other CNS lesions or research, the result of imaging following trauma, or in-

ABBREVIATIONS EOR = extent of resection; FLAIR = fluid attenuation inversion recovery; iLGG = incidental low-grade glioma; KPS = Karnofsky Performance Status; LGG = low-grade glioma; MPFS = malignant progression-free survival; OS = overall survival; $P F S=$ progression-free survival; $R O I=$ region of interest; $S D=$ standard deviation; SEM = standard error of the mean.

SUBMITTED January 17, 2017. ACCEPTED March 17, 2017.

INCLUDE WHEN CITING Published online October 6, 2017; DOI: 10.3171/2017.3.JNS17159. 
vestigation for nonspecific symptoms. Infiltrating gliomas are an important group of incidental lesions discovered on brain imaging due to the significant impact they will ultimately impose on individuals who harbor them.

Growing evidence in the literature supports the significantly positive impact of maximal safe resection on overall survival (OS) in patients with symptomatic glioma. The management of incidental gliomas in asymptomatic patients remains controversial, posing a significant dilemma to both the surgeon and the patient. The poorly understood natural history of these lesions and paucity of literature about these tumors contribute to this dilemma. Many neurosurgeons adopt a "watch-and-wait" approach ${ }^{19,22}$ and intervene only when clinical symptoms manifest or there is radiological evidence of disease progression. A growing number of neurosurgeons with special interest in neurooncology, however, believe that early resection allows for greater extent of resection resulting in longer survival., ${ }^{111}$ Pallud et al. have demonstrated that surgical excision of incidental low-grade gliomas (iLGGs) increases survival. ${ }^{19}$ Also, in their most recent case report, Cochereau et al. illustrate the fact that there can be acute malignant transformation of iLGGs even when the patient remains symptom free. ${ }^{7}$ We set out to review a series of cases of iLGGs treated during a period when the general approach at our institution was "watch-and-wait" to determine the characteristics of these tumors and treatment outcomes.

\section{Methods}

This study was approved by the Health Research Ethics Board of Alberta Cancer Committee at the University of Calgary.

\section{Patient Selection}

We retrospectively identified adult patients (age $\geq 18$ years) who underwent surgery at the Foothills Medical Center for low-grade infiltrative glioma between April 2004 and April 2016 and had at least 6 months of postoperative follow-up. Surgery was performed by 8 different neurosurgeons. Patient electronic charts, including outpatient neuro-oncology notes, were reviewed for clinical presenting history, radiological images, operative report, and hospital course. Patients with incidentally discovered LGG were selected. An "incidental" glioma is defined in this study as the finding of a glioma on brain imaging that was obtained for reasons or neurological symptoms not attributable to the tumor, such as trauma, headache without associated mass effect or sign of intracranial hypertension, screening for other neurological diseases, or research purposes. Histopathological diagnosis and tumor grade based on 2007 WHO guidelines as well as molecular genetic profiles (including MGMT, IDH, ATRX, P53, and $1 \mathrm{p} 19 \mathrm{q}$ co-deletion status) were noted from medical records. WHO Grade II was confirmed for all patients included in the study. Patients who were symptomatic or had high-grade pathology (based on WHO criteria) were excluded.

\section{Volumetric Analysis}

Volumetric analysis of tumor volume, growth rate, and extent of resection (EOR) was performed by the first author (M.O.D.). Using the Myrian Community 2.0.0 software (Intrasense), regions of interest (ROIs) were outlined on axial fluid attenuated inversion recovery (FLAIR) or T2-weighted images (starting and finishing on the outermost axial slices-i.e., superior and inferior, proximal or distal) to measure tumor volumes on diagnostic, preoperative, and postoperative MRI studies. Postoperative MRI was performed within 24-48 hours of surgery. Areas on the postoperative imaging with increased FLAIR or T2 signal with extension into areas with no signal on the preoperative MRI were presumed to be postsurgical changes not included in the ROI. Extent of resection was computed as ([(preoperative tumor volume - postoperative volume)/ preoperative tumor volume] $\times 100$ ). Tumor growth rate $\left(\mathrm{cm}^{3} /\right.$ month) was calculated as (mean preoperative tumor volume - mean diagnostic tumor volume)/time from diagnosis to resection.

\section{Primary Outcome Measure}

Outcome measures were assessed and defined as follows. Overall survival (OS) was defined as time from initial resection to death. Progression-free survival (PFS) was defined as time from initial resection to evidence of tumor enlargement on follow-up MRI. Malignant progressionfree survival (MPFS) was defined as time from initial resection to evidence of contrast enhancement on follow-up MRI or higher-grade histopathology from subsequent surgery. Patients with known progression and/or malignant transformation or death were censored as of their last MRI date or last clinic follow-up date, respectively.

\section{Statistical Analysis}

Statistical analysis was performed using GraphPad Prism 7 software. Frequency distribution and summary statistics were calculated for all variables. For categorical variables, cross-tabulation was generated and Wilcoxon and Fisher tests were used to compare distributions. Statistical significance was defined as $p<0.05$. Survival (OS, PFS, and MPFS) was estimated using the Kaplan-Meier method. Results of Kaplan-Meier analysis are reported as means with standard error of the mean (SEM). Comparison of survival curves according to various parameters was performed using the Mantel-Cox test. A univariate Cox proportional hazards model was used to assess the prognostic significance of multiple variables with respect to survival.

\section{Results}

\section{Patient Demographics}

A total of 501 patients with newly diagnosed LGG were treated at our institution between April 2004 and April 2016. Thirty-four patients $(6.8 \%)$ from this population met the study inclusion criteria. Characteristics of these $34 \mathrm{pa}-$ tients are summarized in Table 1 . The group included 17 men and 17 women, and their mean age at tumor discovery was 40.8 years (standard deviation [SD] 12.5 years). Headache was the most common indication for brain imaging leading to the diagnosis of glioma $(26 \%, \mathrm{n}=9)$, followed closely by screening $(21 \%, \mathrm{n}=7)$. Other reasons 
TABLE 1. Clinical and demographic characteristics of patients with iLGG

\begin{tabular}{|c|c|}
\hline Characteristic & Value \\
\hline No. of patients & 34 \\
\hline \multicolumn{2}{|l|}{ Age at diagnosis (yrs) } \\
\hline Mean & 40.8 \\
\hline SD & 12.5 \\
\hline Range & $20-63$ \\
\hline \multicolumn{2}{|l|}{ Sex } \\
\hline Male & $17(50 \%)$ \\
\hline Female & $17(50 \%)$ \\
\hline \multicolumn{2}{|l|}{ KPS score at diagnosis } \\
\hline 90 & $12(35 \%)$ \\
\hline 100 & $22(65 \%)$ \\
\hline \multicolumn{2}{|l|}{ Mode of discovery } \\
\hline Headaches & $9(26 \%)$ \\
\hline Screening & $7(21 \%)$ \\
\hline Trauma & $5(15 \%)$ \\
\hline Syncope & $3(8.8 \%)$ \\
\hline Dizziness & $3(8.8 \%)$ \\
\hline Hearing loss & $2(5.7 \%)$ \\
\hline Psychosis & $1(2.9 \%)$ \\
\hline Other & $4(11.8 \%)$ \\
\hline \multicolumn{2}{|l|}{ Side of tumor } \\
\hline Right & $19(56 \%)$ \\
\hline Left & $15(44 \%)$ \\
\hline \multicolumn{2}{|l|}{ Tumor location } \\
\hline Frontal & 18 \\
\hline Temporal & 5 \\
\hline Parietal & 3 \\
\hline Parieto-occipital & 3 \\
\hline Insular & 3 \\
\hline Opercular & 1 \\
\hline Thalamic & 1 \\
\hline \multicolumn{2}{|c|}{ Tumor vol at diagnosis $\left(\mathrm{cm}^{3}\right)$} \\
\hline Mean & 36.8 \\
\hline SD & 42.5 \\
\hline Range & $2.31-169$ \\
\hline Median & 18.8 \\
\hline IQR & $5.8-45.6$ \\
\hline \multicolumn{2}{|l|}{ Preop tumor vol $\left(\mathrm{cm}^{3}\right)$} \\
\hline Mean & 57.2 \\
\hline SD & 62.2 \\
\hline Range & $5.89-216$ \\
\hline Median & 35.5 \\
\hline IQR & $7.2-92.5$ \\
\hline \multicolumn{2}{|l|}{ Time to resection (mos) } \\
\hline Mean & 27.6 \\
\hline SD & 39.2 \\
\hline Range & $0-161$ \\
\hline Median & 11.5 \\
\hline IQR & $2.0-35.5$ \\
\hline
\end{tabular}

» CONTINUED FROM PREVIOUS COLUMN

TABLE 1. Clinical and demographic characteristics of patients with iLGG

\begin{tabular}{cc}
\hline \multicolumn{1}{c}{ Characteristic } & Value \\
\hline Extent of resection & \\
\hline $100 \%$ & 8 \\
\hline $75-99 \%$ & 11 \\
\hline $50-74 \%$ & 9 \\
\hline $0-49 \%$ & 6 \\
\hline Mean & $81.03 \%$ \\
\hline SD & $19.9 \%$ \\
\hline Median & $85.4 \%$ \\
\hline IQR & $63.3-100 \%$ \\
\hline Clinical follow-up (yrs) & 5.02 \\
\hline Mean & 3.9 \\
\hline SD & $0.5-12$ \\
\hline Range & 4.2 \\
\hline Median & $1.5-9.1$ \\
\hline IQR &
\end{tabular}

for imaging included trauma, syncope, dizziness, hearing loss, acute psychosis, and nonspecific neurological signs that did not localize to the site of the glioma. Screening was done for family history of cerebral aneurysm, moyamoya disease, neurofibromatosis, and multiple sclerosis. One patient was screened following resection of a spinal schwannoma and cytogenetic test confirming neurofibromatosis Type 2 . In patients presenting with headaches, the headaches were usually "migrainous" and long-standing in nature, without changes in character over time. Furthermore, worrisome symptoms such as nausea/vomiting or worsening in the morning, as typically described in headache due to neoplasm, were not present, and imaging typically did not demonstrate vasogenic edema or mass effect. Also, the size of the tumors among iLGG patients presenting with headaches, although variable, was generally smaller at the time of diagnosis than the size of the tumors in the rest of this patient cohort. The mean time from discovery of an incidental glioma to surgery was 27.6 months (range 0-161 months). In general, during this period patients with iLGGs were followed with MRI surveillance and underwent resection once changes were noted on imaging or they became symptomatic. Five patients opted for early resection rather than surveillance, but the remaining patients only underwent resection after evidence of progression was detected on follow-up imaging. Four patients underwent biopsy for definitive diagnosis. No patient had documented neurological deficit preoperatively; the preoperative Karnofsky Performance Status (KPS) score was 100 in 22 patients (65\%) and 90 in $12(35 \%)$.

\section{Tumor Characteristics}

We hypothesized that iLGGs would be smaller in size and located predominantly in nondominant locations. Imaging review demonstrated that the frontal lobe was the most common location $(52.9 \%, \mathrm{n}=18)$ and that 19 tumors 

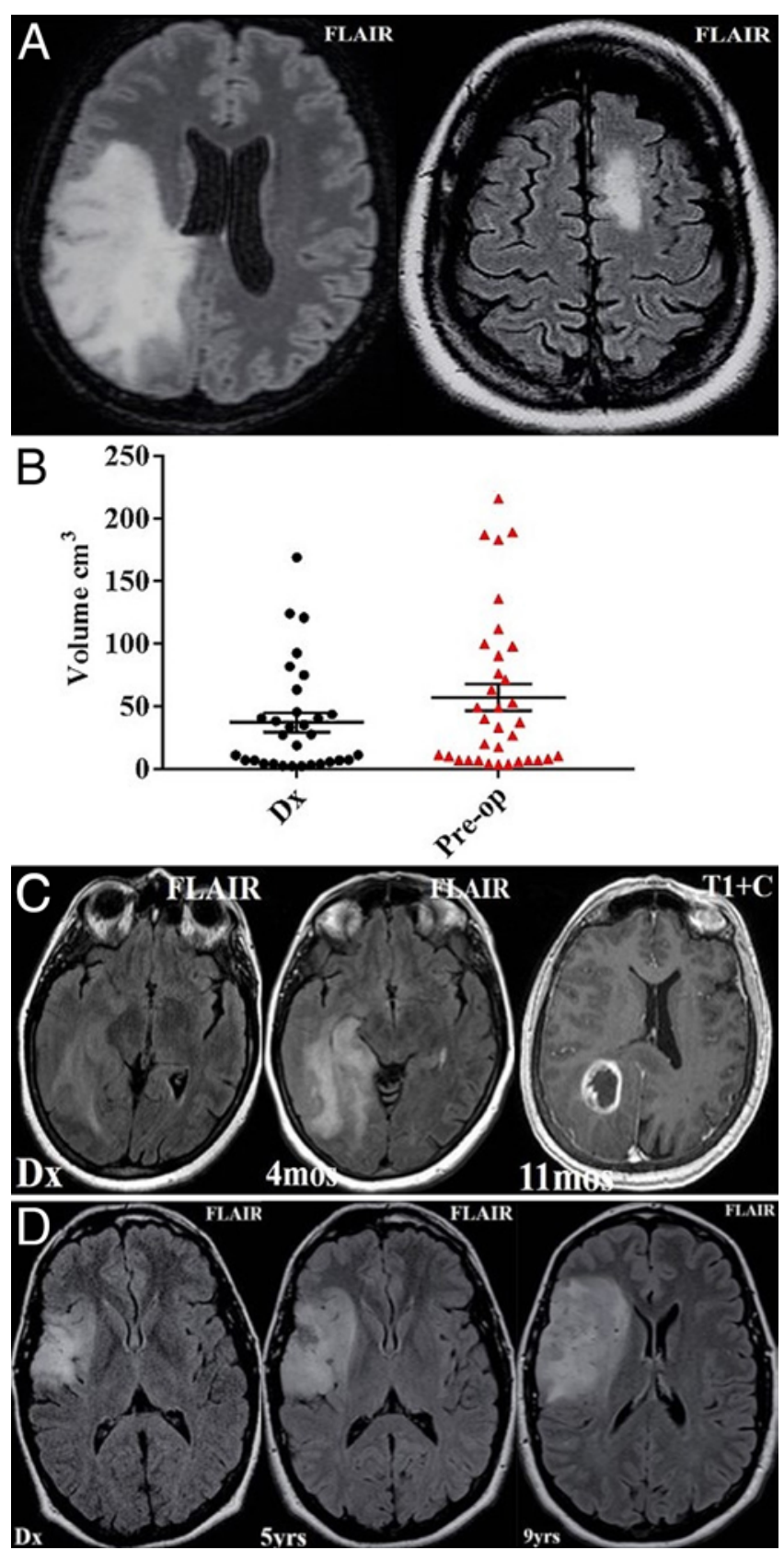

FIG. 1. Imaging characteristics and volumetric assessment of iLGGs. A: Axial FLAIR MR images obtained in 2 different patients at the time of diagnosis of LGG demonstrating the substantial variability of lesion size. B: Scatterplot showing iLGG tumor volumes at the time of diagnosis (Dx) and preoperatively. C and D: Axial FLAIR MR images showing 2 iLGGs with differing natural history and progression. The images in C were obtained in a patient with a rapidly progressive lesion, whereas the images in $\mathrm{D}$ were obtained in a patient with a slowly progressing iLGG. Figure is available in color online only.

(56\%) were lateralized to the nondominant hemisphere. Other locations were temporal, parietal, parieto-occipital, insular, opercular, and thalamic. By performing volumetric analysis with MRI, we evaluated imaging characteristics of iLGGs and their evolution prior to treatment. Surprisingly, tumor size at the time of diagnosis varied widely
TABLE 2. Histopathological characteristics of resected tumors

\begin{tabular}{lc}
\hline \multicolumn{1}{c}{ Tumor Subtype } & No. of Cases \\
\hline Oligodendroglioma & \\
\hline \multicolumn{1}{c}{ Oligodendroglioma, IDH mutant \& 1p19q co-deleted } & 13 \\
\hline Oligodendroglioma, not otherwise specified & 3 \\
\hline Astrocytoma & 12 \\
\hline$\quad$ Diffuse astrocytoma, IDH mutant & 3 \\
\hline$\quad$ Diffuse astrocytoma, IDH wild type & 2 \\
\hline Oligoastrocytoma* & 1 \\
\hline Ganglioglioma*
\end{tabular}

* Molecular genetic testing was not performed in these cases.

(range 2.31-169 $\mathrm{cm}^{3}$ ), as depicted in Fig. 1A. All but 5 patients had a volume increase on preoperative imaging compared with imaging at diagnosis (Fig. 1B). The mean tumor volume at diagnosis was $36.8 \mathrm{~cm}^{3}$, and the mean preoperative volume was $57.2 \mathrm{~cm}^{3}$ (range $5.89-216 \mathrm{~cm}^{3}$ ). Within the patient cohort, there was a wide variation in the rate of change in tumor size, ranging from rapid growth within months (Fig. 1C) to slow growth over several years (Fig. 1D).

\section{Surgical Results}

Other than 2 patients who underwent an awake craniotomy, all patients were operated on under general anesthesia. There was no operative death recorded. Postoperatively 1 patient had transient akinetic mutism, which resolved within 48 hours, prior to discharge from the hospital. Another patient suffered a middle cerebral artery perforator infarct in the capsuloganglionic region, leaving him with mild hemiparesis and dysphasia. The mean postoperative hospital stay was 3 days (range 1-7 days). Eleven patients (32\%) were discharged with a KPS score of 90 , and $23(68 \%)$ were discharged with a score of 100. The EOR was $100 \%$ in 8 cases $(23.5 \%), 75 \%-99 \%$ in $11(32.4 \%), 50 \%-74 \%$ in $9(26.5 \%)$, and $0 \%-49 \%$ in 6 $(17.6 \%)$. Histopathological review confirmed LGG in all 34 cases. All but 2 patients had molecular genetic testing, including assessment of MGMT, IDH, P53, ATRX, and $1 \mathrm{p} 19 \mathrm{q}$ co-deletion status. Based on this knowledge and using the 2016 WHO classification of CNS tumors, 13 patients $(38.2 \%)$ had oligodendroglioma, IDH mutant and 1p19q co-deleted; 3 (8.8\%) had oligodendroglioma, not otherwise specified; $12(35.3 \%)$ had diffuse astrocytoma, IDH mutant; and $3(8.8 \%)$ had diffuse astrocytoma, IDH wild type. No molecular genetic testing was performed in 3 patients; oligoastrocytoma was diagnosed in 2 of these patients and ganglioglioma in 1 patient (Table 2). Sixteen patients $(47 \%)$ developed early ( $<3$ months after surgery) or delayed ( $\geq 3$ months after surgery) postoperative seizure disorder. Patients with diffuse astrocytoma had a higher incidence of developing postoperative seizures than did those with oligodendroglioma (Table 3).

\section{Survival}

The mean duration of follow-up was 5.1 years (range $0.5-12$ years). Twelve patients $(35.3 \%)$ had disease pro- 
TABLE 3. Distribution of postoperative seizures by tumor pathology

\begin{tabular}{cc}
\hline Seizure Timing \& Pathology & Value \\
\hline Early postop seizures & \\
\hline Oligodendroglioma & 1 \\
\hline Astrocytoma & 5 \\
\hline Late postop seizures & \\
\hline Oligodendroglioma & 4 \\
\hline Astrocytoma & 6 \\
\hline
\end{tabular}

gression; the average time to progression in these cases was 43.8 months (range 3-105 months) (Table 4). There were 9 cases of malignant transformation $(26.5 \%)$ and 4 deaths $(11.8 \%)$. All but one of the patients who died in the study had malignant transformation from LGG to glioblastoma; the single exception was a patient who died of Stage IV pancreatic cancer. Eight patients underwent repeat resection, and 3 of these patients received adjuvant temozolomide and/or radiation therapy. Kaplan-Meier estimates of OS, PFS, and MPFS are shown in Fig. 2. The 10 -year survival rate was greater than $80 \%$ (with only 4 deaths, representing $11.8 \%$ of the patient cohort, median survival could not be estimated). The median survival times for PFS and MPFS, however, were approximately 6 and 9 years. Survival (OS, PFS, MPFS) curves were compared according to EOR, histopathology (Fig. 3), and IDH mutation status (Fig. 4). Although no patient with $100 \%$ EOR had died when the data were analyzed, there was no statistically significant association between EOR and OS ( $\mathrm{p}=0.82)$. Oligodendroglioma and $I D H$ mutation were associated with survival benefit $(\mathrm{p}<0.0001)$.

\section{Discussion}

Incidental low-grade glioma (iLGG) is a WHO Grade II glioma discovered on brain imaging performed for a reason not attributable to the lesion. The incidence of
iLGG has been shown to range from $0.025 \%$ to $0.3 \%$ in population-based imaging studies, ${ }^{2,13,18,21,26}$ and thus these lesions account for 3\%-5\% of all LGGs. ${ }^{10,17}$ In our study we found that iLGGs accounted for $6.8 \%$ of cases in our group of patients with LGGs, a similar proportion to that reported in the literature. Furthermore, 94\% of our patients' tumors were oligodendrogliomas and/or astrocytomas. IDH mutation was present in $74 \%$ and was significantly associated with improved survival (OS, PFS, and MPFS). These results are in keeping with results of previous studies evaluating genetics and mutations present in tissues from resected iLGGs. The ubiquitous use of brain imaging technology in the developed world, and increasing access to brain imaging worldwide, will likely lead to a rise in the reported incidence of iLGGs, most of which harbor IDHI mutation. ${ }^{9,17}$ Consequently, neurosurgeons will be increasingly faced with the dilemma of how to care for patients with iLGGs.

Due to the relative rarity of iLGG, there is a paucity of data about the natural history of this disease before symptom onset together with the appropriate treatment approach and its timing. Recent studies have demonstrated that iLGGs grow at a velocity similar to their symptomatic counterparts. ${ }^{7,20}$ Not only do iLGGs grow over time, they may also undergo malignant degeneration even in asymptomatic patients. ${ }^{8,12}$ Our results demonstrate that tumor volumes increase by about $20 \mathrm{~cm}^{3}$ over an average of 28 months, and in 1 extreme case rapid progression with malignant transformation occurred that ultimately led to the patient's demise (Fig. 4). When considering management options, the question of whether patients with iLGG are truly asymptomatic must be addressed. In a recent study of neuropsychological disturbance in patients with iLGG, Cochereau et al. reported that $60 \%$ of the patients in their study had neurocognitive dysfunction, suggesting that patients may actually be symptomatic when more sensitive tests are employed at the time of diagnosis. ${ }^{6}$ Resection for the initial presentation of symptomatic LGG is widely accepted, and maximal safe resection is a crucial factor in

TABLE 4. Characteristics of patients with progressive disease after initial biopsy or resection

\begin{tabular}{|c|c|c|c|c|c|c|c|c|}
\hline $\begin{array}{l}\text { Case } \\
\text { No. }\end{array}$ & $\begin{array}{l}\text { Age (yrs) } \\
\text { Sex }\end{array}$ & 2016 WHO Diagnosis & $\begin{array}{l}\text { Preop Tumor } \\
\text { Vol }\left(\mathrm{cm}^{3}\right)\end{array}$ & $\begin{array}{c}\text { EOR } \\
(\%)\end{array}$ & $\begin{array}{c}\text { Time to } \\
\text { Progression (mos) }\end{array}$ & $\begin{array}{c}\text { Repeat } \\
\text { Resection }\end{array}$ & Chemo & RT \\
\hline 1 & $32, \mathrm{~F}$ & Oligoastrocytoma & 63.5 & 92.99 & 64 & Yes & No & No \\
\hline 2 & $20, M$ & Oligodendroglioma, IDH mutant \& 1p19q co-deleted & 99.9 & Biopsy & 105 & Yes & Yes & Yes \\
\hline 3 & $37, \mathrm{~F}$ & Oligodendroglioma, IDH mutant \& 1p19q co-deleted & 90.6 & 86.42 & 67 & Yes & Yes & Yes \\
\hline 4 & $47, M$ & Oligodendroglioma, not otherwise specified & 33.5 & 85.43 & 24 & Yes & No & No \\
\hline 5 & $34, \mathrm{M}$ & Diffuse astrocytoma, IDH mutant & 3.87 & 100.00 & 32 & No & No & No \\
\hline 6 & $36, F$ & Oligodendroglioma & 20.2 & 63.81 & 24 & Yes & No & No \\
\hline 7 & $55, \mathrm{~F}$ & Oligodendroglioma, IDH mutant \& 1p19q co-deleted & 37.4 & 68.98 & 40 & Yes & No & Yes \\
\hline 8 & $20, F$ & Diffuse astrocytoma, IDH mutant & 7.04 & 76.28 & 45 & Yes & No & No \\
\hline 9 & $31, M$ & Diffuse astrocytoma, IDH mutant & - & Biopsy & 48 & Yes & No & No \\
\hline 10 & $60, \mathrm{~F}$ & Diffuse astrocytoma, IDH wild type & 98.1 & 87.77 & 3 & No & No & No \\
\hline 11 & $47, \mathrm{~F}$ & Diffuse astrocytoma, IDH mutant & 71.4 & 51.82 & 16 & No & Yes & Yes \\
\hline 12 & $46, M$ & Glioblastoma, IDH wild type & 4.11 & 82.97 & 58 & No & No & No \\
\hline
\end{tabular}

Chemo = chemotherapy; RT = radiotherapy . 

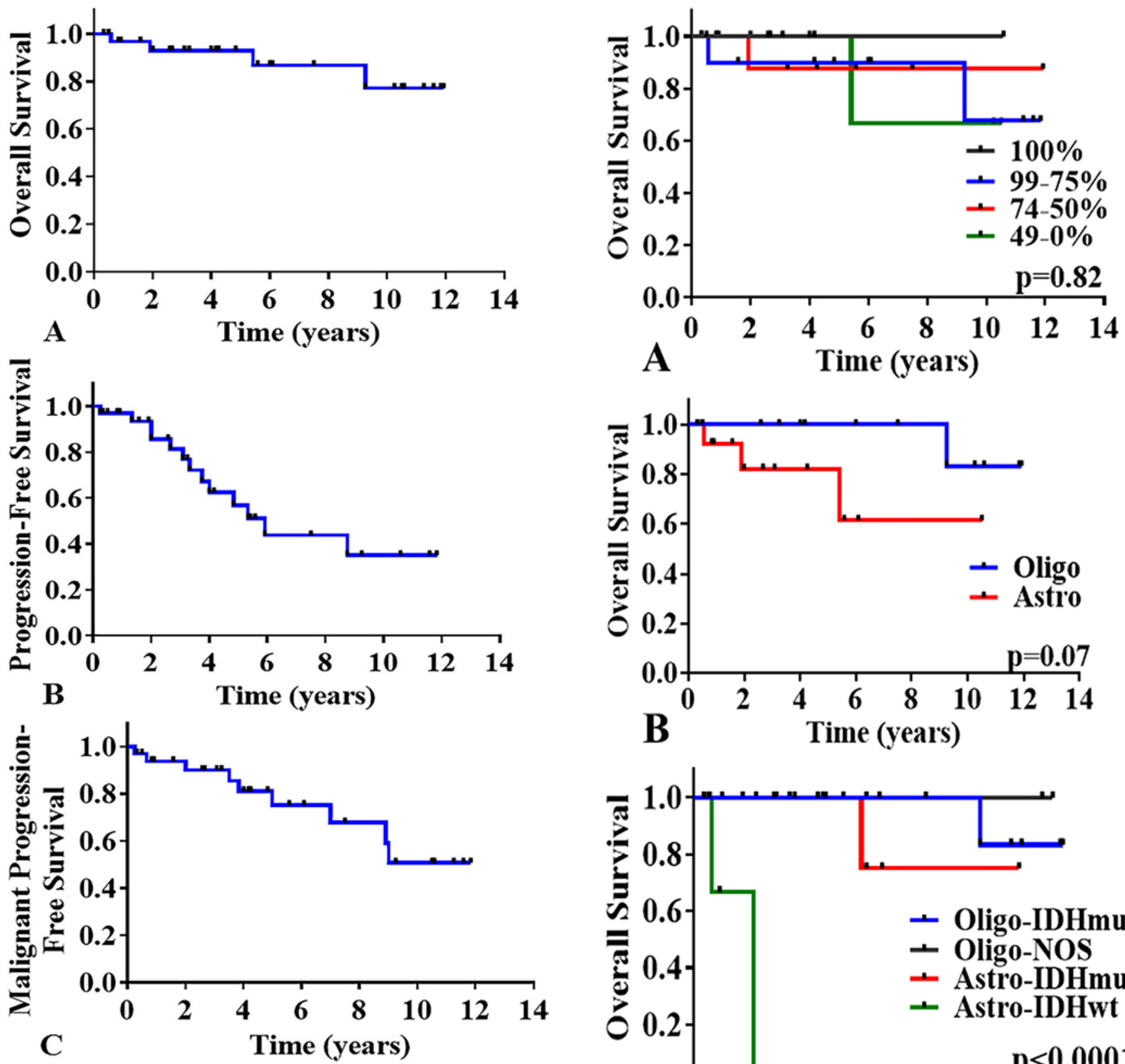

FIG. 2. Kaplan-Meier curves for OS (A), PFS (B), and MPFS (C) in patients with iLGG. Figure is available in color online only.

determining survival. ${ }^{5,14,16,24}$ Incidental LGGs are believed to represent an earlier step in the pathological progression of LGG. Various investigators (Duffau, ${ }^{8}$ Pallud et al.,${ }^{19}$ and Potts et $\mathrm{al}^{20}$ ) have demonstrated that resection of iLGGs leads to an improved survival compared with survival in symptomatic cohorts. This finding was attributable to low tumor volume, noneloquent location, and greater rates of EOR in iLGGs compared with symptomatic LGGs. Our study also showed survival benefit of resection, with $80 \%$ of patients alive at a mean follow-up period of 5 years. About $35 \%$ of the 34 patients included in the study had disease progression, and about $26 \%$ had malignant transformation. Surgery-related complications were minimal,
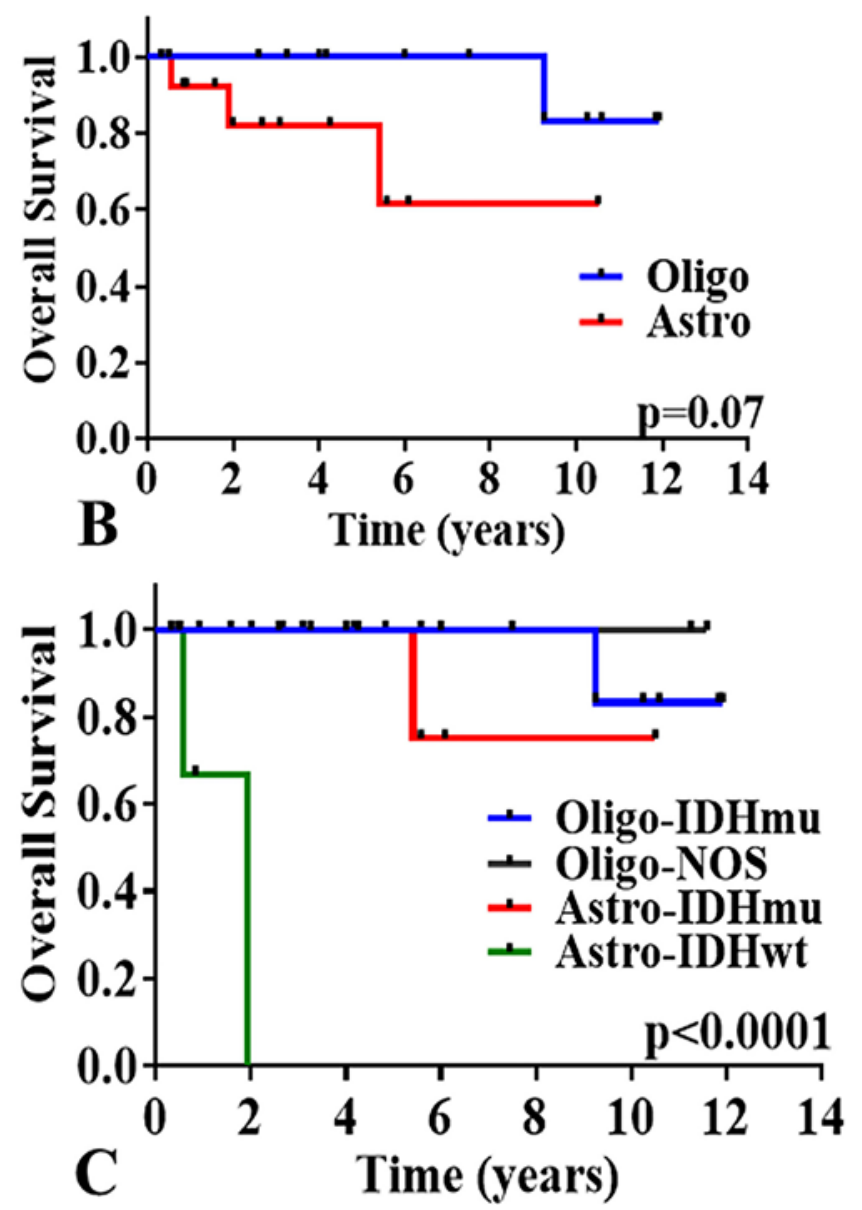

FIG. 3. Correlation of OS with EOR ( $p=0.82$, log-rank test) (A), histopathology ( $p=0.07$, log-rank test) $(B)$, and histopathology and IDH mutation status ( $p<0.0001$, log-rank test) (C). Oligoastrocytoma is categorized as astrocytoma in this graph. The case of ganglioglioma was omitted from this analysis. Astro = astrocytoma; IDHmu = IDH mutant; IDHwt = IDH wild type; NOS = not otherwise specified; Oligo = oligodendroglioma. Figure is available in color online only.

with 2 patients suffering mild nondisabling strokes and another experiencing transient akinetic mutism. In light of these findings, together with recent evidence demonstrating survival benefit for resection of symptomatic LGGs, 

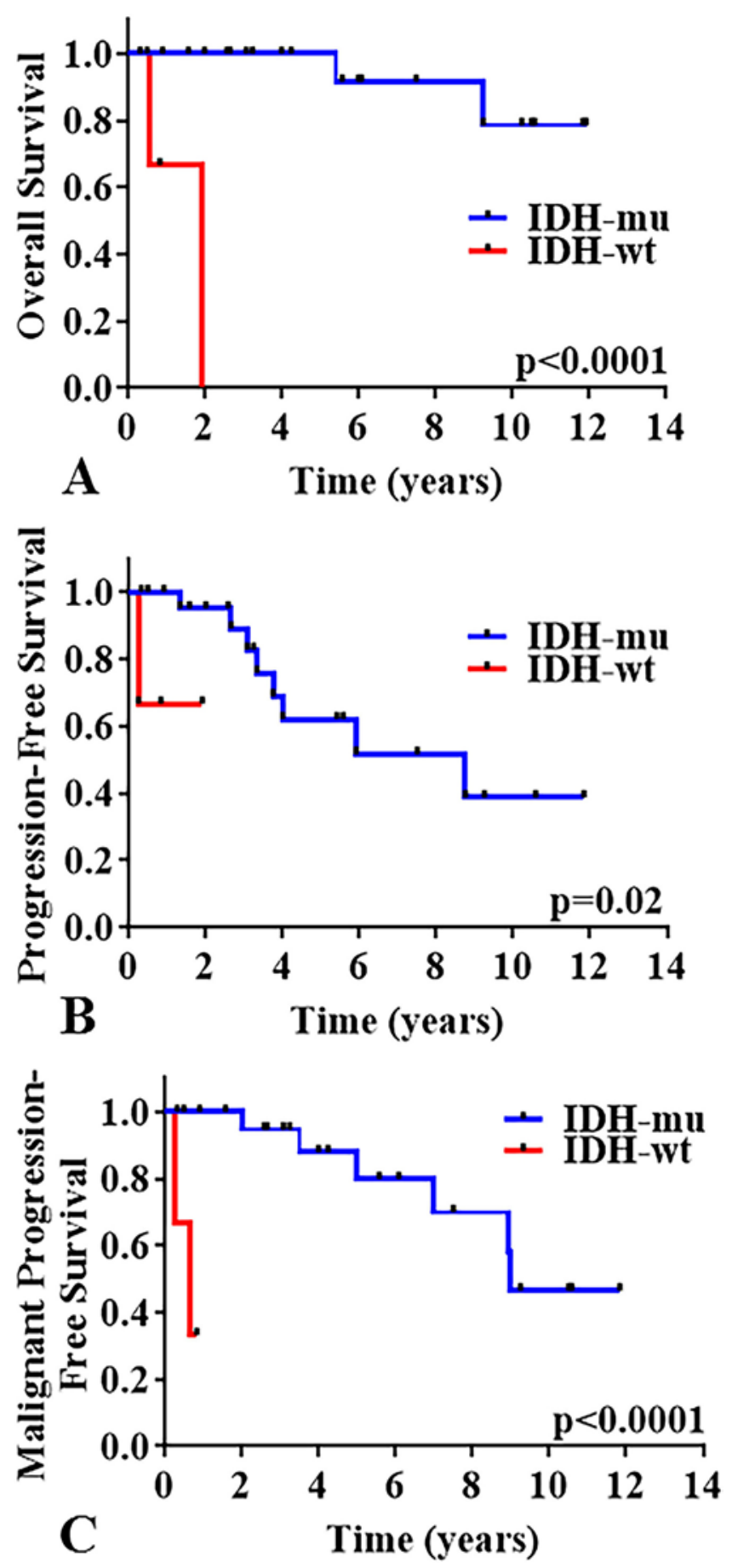

FIG. 4. Association between IDH mutation and survival. Patients with iLGG harboring IDH mutation had significantly improved OS ( $p<0.0001$, log-rank test) $(A)$, longer PFS ( $p=0.02$, log-rank test) (B), and longer MPFS ( $p<0.0001$, log-rank test) (C). Figure is available in color online only.

resective surgery should be considered as a management option for iLGGs.

Seizures are a frequent consequence and initial presenting symptom of LGGs. ${ }^{3,4,23,25}$ Knowledge about the risk of seizures in iLGGs either at presentation or after resection is limited. Lima and Duffau reported seizures in 2 of 21 patients with iLGG who underwent awake surgery..$^{15}$ Our study demonstrated a higher proportion of patients $(16$ [47\%] of 34) who experienced postoperative seizures. In 6 of these patients, the seizures occurred during the early postoperative period ( $<3$ months after surgery) and in 10 they developed during the late postoperative period $(>$ 3 months after surgery). Patients with astrocytic tumors were more likely to develop seizures. These postoperative seizures could be explained by recurrent disease and may simply relate to the natural history of the disease or could perhaps be a functional consequence of resection.

The main limitations of this study are the small sample size and small number of events (i.e., deaths) recorded. Both are due to the rarity of iLGGs. Also due to the limitation of our study design, comparative efficacy of early surgery in iLGGs could not be assessed. Our study failed to demonstrate a statistically significant survival benefit with greater EOR and histopathology, contrary to current knowledge about LGGs, although at the time of manuscript preparation there was no recorded death of any of our patients in whom an EOR of $100 \%$ was achieved. We believe these results reflect the small sample size captured by our study.

\section{Conclusions}

We demonstrate that iLGGs are not quiescent, even in patients thought to remain asymptomatic while harboring these lesions. Importantly, resection can be safely performed without significant consequence to patient performance status and should be favored over a "watchand-wait" approach. Both early and delayed postoperative seizures occur frequently in patients with iLGGs and may be a consequence of treatment or may simply reflect the natural history of LGG. The majority of iLGGs harbor $I D H 1$ mutation, leading to delayed disease progression, longer time before malignant transformation, and improved OS. Balancing early surgical intervention with the principle of primum non nocere should be a primary consideration when individualizing treatment for patients diagnosed with iLGGs.

\section{Acknowledgments}

We would like to thank Joseph E. Amuah, PhD, Canadian Institute for Health Information, for reviewing the statistical analysis in this paper.

\section{References}

1. Aghi MK, Nahed BV, Sloan AE, Ryken TC, Kalkanis SN, Olson JJ: The role of surgery in the management of patients with diffuse low grade glioma: A systematic review and evidence-based clinical practice guideline. J Neurooncol 125:503-530, 2015

2. Bauchet L, Rigau V, Mathieu-Daudé H, Figarella-Branger D, Hugues D, Palusseau L, et al: French brain tumor data bank: methodology and first results on 10,000 cases. J Neurooncol 84:189-199, 2007

3. Beaumont A, Whittle IR: The pathogenesis of tumour associated epilepsy. Acta Neurochir (Wien) 142:1-15, 2000

4. Berger MS, Ghatan S, Haglund MM, Dobbins J, Ojemann GA: Low-grade gliomas associated with intractable epilepsy: 
seizure outcome utilizing electrocorticography during tumor resection. J Neurosurg 79:62-69, 1993

5. Berger MS, Rostomily RC: Low grade gliomas: functional mapping resection strategies, extent of resection, and outcome. J Neurooncol 34:85-101, 1997

6. Cochereau J, Herbet G, Duffau H: Patients with incidental WHO Grade II glioma frequently suffer from neuropsychological disturbances. Acta Neurochir (Wien) 158:305-312, 2016

7. Cochereau J, Herbet G, Rigau V, Duffau H: Acute progression of untreated incidental WHO Grade II glioma to glioblastoma in an asymptomatic patient. J Neurosurg 124:141-145, 2016

8. Duffau H: Awake surgery for incidental WHO Grade II gliomas involving eloquent areas. Acta Neurochir (Wien) 154:575-584, 2012

9. Eskandary H, Sabba M, Khajehpour F, Eskandari M: Incidental findings in brain computed tomography scans of 3000 head trauma patients. Surg Neurol 63:550-553, 2005

10. Floeth FW, Sabel M, Stoffels G, Pauleit D, Hamacher K, Steiger HJ, et al: Prognostic value of 18F-fluoroethyl-L-tyrosine PET and MRI in small nonspecific incidental brain lesions. J Nucl Med 49:730-737, 2008

11. Jakola AS, Myrmel KS, Kloster R, Torp SH, Lindal S, Unsgård $\mathrm{G}$, et al: Comparison of a strategy favoring early surgical resection vs a strategy favoring watchful waiting in lowgrade gliomas. JAMA 308:1881-1888, 2012

12. Kamiguchi H, Shiobara R, Toya S: Accidentally detected brain tumors: clinical analysis of a series of 110 patients. Clin Neurol Neurosurg 98:171-175, 1996

13. Katzman GL, Dagher AP, Patronas NJ: Incidental findings on brain magnetic resonance imaging from 1000 asymptomatic volunteers. JAMA 282:36-39, 1999

14. Keles GE, Chang EF, Lamborn KR, Tihan T, Chang CJ, Chang SM, et al: Volumetric extent of resection and residual contrast enhancement on initial surgery as predictors of outcome in adult patients with hemispheric anaplastic astrocytoma. J Neurosurg 105:34-40, 2006

15. Lima GL, Duffau H: Is there a risk of seizures in "preventive" awake surgery for incidental diffuse low-grade gliomas? J Neurosurg 122:1397-1405, 2015

16. McGirt MJ, Chaichana KL, Attenello FJ, Weingart JD, Than $\mathrm{K}$, Burger PC, et al: Extent of surgical resection is independently associated with survival in patients with hemispheric infiltrating low-grade gliomas. Neurosurgery 63:700-708, 2008

17. Morris Z, Whiteley WN, Longstreth WT Jr, Weber F, Lee YC, Tsushima Y, et al: Incidental findings on brain magnetic resonance imaging: systematic review and meta-analysis. BMJ 339:b3016, 2009

18. Onizuka M, Suyama K, Shibayama A, Hiura T, Horie N, Miyazaki $\mathrm{H}$ : Asymptomatic brain tumor detected at brain checkup. Neurol Med Chir (Tokyo) 41:431-435, 2001
19. Pallud J, Fontaine D, Duffau H, Mandonnet E, Sanai N, Taillandier L, et al: Natural history of incidental World Health Organization grade II gliomas. Ann Neurol 68:727-733, 2010

20. Potts MB, Smith JS, Molinaro AM, Berger MS: Natural history and surgical management of incidentally discovered lowgrade gliomas. J Neurosurg 116:365-372, 2012

21. Recht LD, Lew R, Smith TW: Suspected low-grade glioma: is deferring treatment safe? Ann Neurol 31:431-436, 1992

22. Rees J, Watt H, Jäger HR, Benton C, Tozer D, Tofts P, et al: Volumes and growth rates of untreated adult low-grade gliomas indicate risk of early malignant transformation. Eur J Radiol 72:54-64, 2009

23. Rudà R, Bello L, Duffau H, Soffietti R: Seizures in low-grade gliomas: natural history, pathogenesis, and outcome after treatments. Neuro Oncol 14 (Suppl 4):iv55-iv64, 2012

24. Smith JS, Chang EF, Lamborn KR, Chang SM, Prados MD, Cha $\mathrm{S}$, et al: Role of extent of resection in the long-term outcome of low-grade hemispheric gliomas. J Clin Oncol 26:1338-1345, 2008

25. Smits A, Duffau H: Seizures and the natural history of World Health Organization Grade II gliomas: a review. Neurosurgery 68:1326-1333, 2011

26. Weber F, Knopf H: Incidental findings in magnetic resonance imaging of the brains of healthy young men. J Neurol Sci 240:81-84, 2006

\section{Disclosures}

The authors report no conflict of interest concerning the materials or methods used in this study or the findings specified in this paper.

\section{Author Contributions}

Conception and design: Opoku-Darko, Cairncross, Kelly. Acquisition of data: Opoku-Darko, Lang, Artindale, Kelly. Analysis and interpretation of data: Opoku-Darko, Lang, Sevick. Drafting the article: Opoku-Darko, Kelly. Critically revising the article: Opoku-Darko, Kelly. Reviewed submitted version of manuscript: Opoku-Darko, Kelly. Approved the final version of the manuscript on behalf of all authors: Opoku-Darko. Statistical analysis: Opoku-Darko, Lang. Administrative/technical/material support: Opoku-Darko, Artindale, Sevick, Kelly. Study supervision: Cairncross, Kelly.

\section{Correspondence}

Michael Opoku-Darko, Foothills Medical Center, 1403 29th St. NW, Calgary, Alberta T2N 2T9, Canada. email: mopokuda@ ucalgary.ca. 